\title{
THE KRONECKER PRODUCT OF SCHUR FUNCTIONS INDEXED BY TWO-ROW SHAPES OR HOOK SHAPES.
}

\author{
MERCEDES H. ROSAS
}

\begin{abstract}
The Kronecker product of two Schur functions $s_{\mu}$ and $s_{\nu}$, denoted by $s_{\mu} * s_{\nu}$, is the Frobenius characteristic of the tensor product of the irreducible representations of the symmetric group corresponding to the partitions $\mu$ and $\nu$. The coefficient of $s_{\lambda}$ in this product is denoted by $\gamma_{\mu \nu}^{\lambda}$, and corresponds to the multiplicity of the irreducible character $\chi^{\lambda}$ in $\chi^{\mu} \chi^{\nu}$.

We use Sergeev's Formula for a Schur function of a difference of two alphabets and the comultiplication expansion for $s_{\lambda}[X Y]$ to find closed formulas for the Kronecker coefficients $\gamma_{\mu \nu}^{\lambda}$ when $\lambda$ is an arbitrary shape and $\mu$ and $\nu$ are hook shapes or two-row shapes.

Remmel [9, 10] and Remmel and Whitehead [11] derived some closed formulas for the Kronecker product of Schur functions indexed by two-row shapes or hook shapes using a different approach. We believe that the approach of this paper is more natural. The formulas obtained are simpler and reflect the symmetry of the Kronecker product.
\end{abstract}

\section{INTRODUCTION}

The aim of this paper is to derive an explicit formula for the Kronecker coefficients corresponding to partitions of certain shapes. The Kronecker coefficients, $\gamma_{\mu \nu}^{\lambda}$, arise when expressing a Kronecker product (also called inner or internal product), $s_{\mu} * s_{\nu}$, of Schur functions in the Schur basis,

$$
s_{\mu} * s_{\nu}=\sum_{\mu, \nu} \gamma_{\mu \nu}^{\lambda} s_{\lambda} .
$$

These coefficients can also be defined as the multiplicities of the irreducible representations in the tensor product of two irreducible representations of the symmetric group. A third way to define them is by the comultiplication expansion. Given two alphabets $X=\left\{x_{1}, x_{2}, \cdots\right\}$ and $Y=\left\{y_{1}, y_{2}, \cdots\right\}$

$$
s_{\lambda}[X Y]=\sum_{\mu, \nu} \gamma_{\mu \nu}^{\lambda} s_{\mu}[X] s_{\nu}[Y],
$$

where $s_{\lambda}[X Y]$ means $s_{\lambda}\left(x_{1} y_{1}, x_{1} y_{2}, \cdots, x_{i} y_{j}, \cdots\right)$. Remmel 9, 10] and Remmel and Whitehead [11] have studied the Kronecker product of Schur functions corresponding to two two-row shapes, two hook shapes, and a hook shape and a 
two-row shape. We will use the comultiplication expansion (2) for the Kronecker coefficients, and a formula for expanding a Schur function of a difference of two alphabets due to Sergeev [1] to obtain similar results in a simpler way. We believe that the formulas obtained using this approach are elegant and reflect the symmetry of the Kronecker product. In the three cases we found a way to express the Kronecker coefficients in terms of regions and paths in $\mathbf{N}^{2}$.

\section{BASIC DEFINITIONS}

A partition $\lambda$ of a positive integer $n$, written as $\lambda \vdash n$, is an unordered sequence of natural numbers adding to $n$. We write $\lambda$ as $\lambda=\left(\lambda_{1}, \lambda_{2}, \cdots, \lambda_{n}\right)$, where $\lambda_{1} \geq$ $\lambda_{2} \geq \cdots$, and consider two such strings equal if they differ by a string of zeroes. The nonzero numbers $\lambda_{i}$ are called the parts of $\lambda$, and the number of parts is called the length of $\lambda$, denoted by $l(\lambda)$. In some cases, it is convenient to write $\lambda=\left(1^{d_{1}} 2^{d_{2}} \cdots n^{d_{n}}\right)$ for the partition of $n$ that has $d_{i}$ copies of $i$. Using this notation, we define the integer $z_{\lambda}$ to be $1^{d_{1}} d_{1} ! 2^{d_{2}} d_{2} ! \cdots n^{d_{n}} d_{n}$ !.

We identify $\lambda$ with the set of points $(i, j)$ in $\mathbf{N}^{2}$ defined by $1 \leq j \leq \lambda_{i}$, and refer to them as the Young diagram of $\lambda$. The Young diagram of a partition $\lambda$ is thought of as a collection of boxes arranged using matrix coordinates. For instance, the Young diagram corrresponding to $\lambda=(4,3,1)$ is

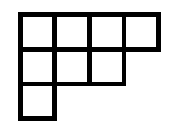

To any partition $\lambda$ we associate the partition $\lambda^{\prime}$, its conjugate partition, defined by $\lambda_{i}^{\prime}=\left|\left\{j: \lambda_{j} \geq i\right\}\right|$. Geometrically, $\lambda^{\prime}$ can be obtained from $\lambda$ by flipping the Young diagram of $\lambda$ around its main diagonal. For instance, the conjugate partition of $\lambda$ is $\lambda^{\prime}=(3,2,2,1)$, and the corresponding Young diagram is

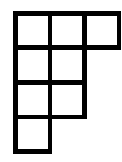

We recall some facts about the theory of representations of the symmetric group, and about symmetric functions. See [7] or [12] for proofs and details.

Let $R\left(S_{n}\right)$ be the space of class function in $S_{n}$, the symmetric group on $n$ letters, and let $\Lambda^{n}$ be the space of homogeneous symmetric functions of degree $n$. A basis for $R\left(S_{n}\right)$ is given by the characters of the irreducible representations of $S_{n}$. Let $\chi^{\mu}$ be the irreducible character of $S_{n}$ corresponding to the partition $\mu$. There is a scalar product $\langle,\rangle_{S_{n}}$ on $R\left(S_{n}\right)$ defined by

$$
\left\langle\chi^{\mu}, \chi^{\nu}\right\rangle_{S_{n}}=\frac{1}{n !} \sum_{\sigma \in S_{n}} \chi^{\mu}(\sigma) \chi^{\nu}(\sigma)
$$


and extended by linearity.

A basis for the space of symmetric functions is given by the Schur functions. There exists a scalar product $\langle,\rangle_{\Lambda^{n}}$ on $\Lambda^{n}$ defined by

$$
\left\langle s_{\lambda}, s_{\mu}\right\rangle_{\Lambda^{n}}=\delta_{\lambda \mu}
$$

where $\delta_{\lambda \mu}$ is the Kronecker delta, and extended by linearity.

Let $p_{\mu}$ be the power sum symmetric function corresponding to $\mu$, where $\mu$ is a partition of $n$. There is an isometry $\operatorname{ch}^{n}: R\left(S_{n}\right) \mapsto \Lambda^{n}$, given by the characteristic map,

$$
\operatorname{ch}^{n}(\chi)=\sum_{\mu \vdash n} z_{\mu}^{-1} \chi(\mu) p_{\mu} .
$$

This map has the remarkable property that if $\chi^{\lambda}$ is the irreducible character of $S_{n}$ indexed by $\lambda$, then $\operatorname{ch}^{n}\left(\chi^{\lambda}\right)=s_{\lambda}$, the Schur function corresponding to $\lambda$. In particular, we obtain that $s_{\lambda}=\sum_{\mu \vdash n} z_{\mu}^{-1} \chi^{\lambda}(\mu) p_{\mu}$. Hence,

$$
\chi^{\lambda}(\mu)=\left\langle s_{\lambda}, p_{\mu}\right\rangle \text {. }
$$

Let $\lambda, \mu$, and $\nu$ be partitions of $n$. The Kronecker coefficients $\gamma_{\mu \nu}^{\lambda}$ are defined by

$$
\gamma_{\mu \nu}^{\lambda}=\left\langle\chi^{\lambda}, \chi^{\mu} \chi^{\nu}\right\rangle_{S_{n}}=\frac{1}{n !} \sum_{\sigma \in S_{n}} \chi^{\lambda}(\sigma) \chi^{\mu}(\sigma) \chi^{\nu}(\sigma) .
$$

Equation (4) shows that the Kronecker coefficients $\gamma_{\mu \nu}^{\lambda}$ are symmetric in $\lambda, \mu$, and $\nu$. The relevance of the Kronecker coefficients comes from the following fact: Let $X^{\mu}$ be the representation of the symmetric group corresponding to the character $\chi^{\mu}$. Then $\chi^{\mu} \chi^{\nu}$ is the character of $X^{\mu} \otimes X^{\nu}$, the representation obtained by taking the tensor product of $X^{\mu}$ and $X^{\nu}$. Moreover, $\gamma_{\mu \nu}^{\lambda}$ is the multiplicity of $X^{\lambda}$ in $X^{\mu} \otimes X^{\nu}$.

Let $f$ and $g$ be homogeneous symmetric functions of degree $n$. The Kronecker product, $f * g$, is defined by

$$
f * g=\operatorname{ch}^{n}(u v)
$$

where $\operatorname{ch}^{n} u=f, \operatorname{ch}^{n} v=g$, and $u v(\sigma)=u(\sigma) v(\sigma)$. To obtain (1) from this definition, we set $f=s_{\mu}, g=s_{\nu}, u=\chi^{\mu}$, and $v=\chi^{\nu}$ in (5).

The Kronecker product has the following symmetries:

$$
\begin{aligned}
& s_{\mu} * s_{\nu}=s_{\nu} * s_{\mu} . \\
& s_{\mu} * s_{\nu}=s_{\mu^{\prime}} * s_{\nu^{\prime}} .
\end{aligned}
$$

Moreover, if $\lambda$ is a one-row shape

$$
\gamma_{\mu \nu}^{\lambda}=\delta_{\mu, \nu}
$$


We introduce the operation of substitution or plethysm into a symmetric function. Let $f$ be a symmetric function, and let $X=\left\{x_{1}, x_{2}, \cdots\right\}$ be an alphabet. We write $X=x_{1}+x_{2}+\cdots$, and define $f[X]$ by,

$$
f[X]=f\left(x_{1}, x_{2}, \cdots\right) .
$$

In general, if $u$ is any element of $\mathbf{Q}\left[\left[x_{1}, x_{2}, \cdots\right]\right]$, we write $u$ as $\sum_{\alpha} c_{\alpha} u_{\alpha}$ where $u_{\alpha}$ is a monomial with coefficient 1 . Then $p_{\lambda}[u]$ is defined by setting

$$
\begin{aligned}
& p_{n}[u]=\sum_{\alpha} c_{\alpha} u_{\alpha}^{n} \\
& p_{\lambda}[u]=p_{\lambda_{1}}[u] \cdots p_{\lambda_{n}}[u]
\end{aligned}
$$

for $\lambda=\left(\lambda_{1}, \cdots, \lambda_{n}\right)$. We define $f[u]$ for all symmetric functions $f$ by saying that $f[u]$ is linear in $f$.

The operation of substitution into a symmetric function has the following properties. For $\alpha$ and $\beta$ rational numbers, $(\alpha f+\beta g)[u]=\alpha f[u]+\beta g[u]$. Moreover, if $c_{\alpha}=1$ for all $\alpha$, then $f[u]=f\left(\cdots, u_{\alpha}, \cdots\right)$.

Let $X=x_{1}+x_{2}+\cdots$ and $Y=y_{1}+y_{2}+\cdots$ be two alphabets. Define the sum of two alphabets by $X+Y=x_{1}+x_{2}+\cdots+y_{1}+y_{2}+\cdots$, and the product of two alphabets by $X Y=x_{1} y_{1}+\cdots+x_{i} y_{j}+\cdots$. Then

$$
\begin{aligned}
p_{n}[X+Y] & =p_{n}[X]+p_{n}[Y], \\
p_{n}[X Y] & =p_{n}[X] p_{n}[Y] .
\end{aligned}
$$

The inner product of function in the space of symmetric functions in two infinite alphabets is defined by

$$
\langle,\rangle_{X Y}=\langle,\rangle_{X}\langle,\rangle_{Y}
$$

where for any given alphabet $Z,\langle,\rangle_{Z}$ denotes the inner product of the space of symmetric functions in $Z$.

For all partitions $\rho$, we have that $p_{\rho}[X Y]=p_{\rho}[X] p_{\rho}[Y]$. If we rewrite (3) as $p_{\rho}=\sum_{\lambda} \chi^{\lambda}(\rho) s_{\lambda}$, then

$$
\sum_{\lambda} \chi^{\lambda} s_{\lambda}[X Y]=\sum_{\mu, \nu} \chi^{\mu} \chi^{\nu} s_{\mu}[X] s_{\nu}[Y]
$$

Taking the coefficient of $\chi^{\lambda}$ on both sides of the previous equation we obtain

$$
s_{\lambda}[X Y]=\sum\left\langle\chi^{\lambda}, \chi^{\mu} \chi^{\nu}\right\rangle s_{\mu}[X] s_{\nu}[Y]
$$

Finally, using the definition of Kronecker coefficients (4) we obtain the comultiplication expansion (2). 
Notation. Let $p$ be a point in $\mathbf{N}^{2}$. We say that $(i, j)$ can be reached from $p$, written $p \leadsto(i, j)$, if $(i, j)$ can be reached from $p$ by moving any number of steps south-west or north-west. We define the weight function $\omega$ by

$$
\omega_{p}(i, j)= \begin{cases}x^{i} y^{j}, & \text { if } p \leadsto(i, j), \\ 0, & \text { otherwise. }\end{cases}
$$

In particular, $\sigma_{k, l}(h)=0$ if $h<0$.

Notation. We denote by $\lfloor x\rfloor$ the largest integer less than or equal to $x$ and by $\lceil x\rceil$ the smallest integer greater than or equal to $x$.

If $f$ is a formal power series, then $\left[x^{\alpha}\right] f$ denotes the coefficient of $x^{\alpha}$ in $f$.

Following Donald Knuth we denote the characteristic function applied to a proposition $P$ by enclosing $P$ with brackets,

$$
(P)= \begin{cases}1, & \text { if proposition } P \text { is true } \\ 0, & \text { otherwise. }\end{cases}
$$

\section{THE CASE OF TWO TWO-ROW SHAPES}

The object of this section is to find a closed formula for the Kronecker coefficients when $\mu=\left(\mu_{1}, \mu_{2}\right)$ and $\nu=\left(\nu_{1}, \nu_{2}\right)$ are two-row shapes, and when we do not have any restriction on the partition $\lambda$. We describe the Kronecker coefficients $\gamma_{\mu \nu}^{\lambda}$ in terms of paths in $\mathbf{N}^{2}$. More precisely, we define two rectangular regions in $\mathbf{N}^{2}$ using the parts of $\lambda$. Then we count the number of points in $\mathbf{N}^{2}$ inside each of these rectangles that can be reached from $\left(\nu_{2}, \mu_{2}+1\right)$, if we are allowed to move any number of steps south-west or north-west. Finally, we subtract these two numbers.

We begin by introducing two lemmas that allow us to state Theorem 1 in a concise form.

Notation. We use the coordinate axes as if we were working with matrices with first entry $(0,0)$. That is, the point $(i, j)$ belongs to the $i$ th row and the $j$ th column.

Lemma 1. Let $k$ and $l$ be positive numbers. Let $R$ be the rectangle with width $k$, height $l$, and upper-left square $(0,0)$. Define

$$
\sigma_{k, l}(h)=\left|\left\{(u, v) \in R \cap \mathbf{N}^{2}:(0, h) \sim(u, v)\right\}\right|
$$

Then

$$
\sigma_{k, l}(h)= \begin{cases}0, & \text { if } h<0 \\ \left\lfloor\left(\frac{h}{2}+1\right)^{2}\right\rfloor, & \text { if } 0 \leq h<\min (k, l) \\ \sigma_{k, l}(s)+\left(\frac{h-s}{2}\right) \min (k, l), & \text { if } \min (k, l) \leq h<\max (k, l) \\ \left\lceil\frac{k l}{2}\right\rceil-\sigma_{k, l}(k+l-h-4), & \text { if } h \text { is even and } \max (k, l) \leq h \\ \left\lfloor\frac{k l}{2}\right\rfloor-\sigma_{k, l}(k+l-h-4), & \text { if } h \text { is odd and } \max (k, l) \leq h\end{cases}
$$


where $s$ is defined as follows: If $h-\min (k, l)$ is even, then $s=\min (k, l)-2$; otherwise $s=\min (k, l)-1$.

Proof. If $h$ is to the left of the 0th column, then we cannot reach any of the points in $\mathbf{N}^{2}$ inside $R$. Hence, $\sigma_{k, l}(h)$ should be equal to zero.

If $0 \leq h \leq \min (k, l)$, then we are counting the number of points in $\mathbf{N}^{2}$ that can be reached from $(0, h)$ inside the square $S$ of side $\min (k, l)$. We have to consider two cases. If $h$ is odd, then we are summing $2+4+\cdots+(h+1)=\left\lfloor\left(\frac{h}{2}+1\right)^{2}\right\rfloor$. On the other hand, if $h$ is even, then we are summing $1+3+\cdots+(h+1)=\left(\frac{h}{2}+1\right)^{2}$.

If $\min (k, l) \leq h<\max (k, l)$, then we subdivide our problem into two parts. First, we count the number of points in $\mathbf{N}^{2}$ that can be reached from $(0, h)$ inside the square $S$ by $\sigma_{k, l}(s)$. Then we count those points in $\mathbf{N}^{2}$ that are in $R$ but not in $S$. Since $h<\max (k, l)$ all diagonals have length $\min (k, l)$ and there are $\frac{h-s}{2}$ of them. See Table 1.

If $\max (k, l) \leq h$, then it is easier to count the total number of points in $\mathbf{N}^{2}$ that can be reached from $(0, h)$ inside $R$ by choosing another parameter $\hat{h}$ big enough and with the same parity as $h$. Then we subtract those points in $\mathbf{N}^{2}$ in $R$ that are not reachable from $(0, h)$ because $h$ is too close. If $h$ is even this number is $\lceil k l / 2\rceil$. If $h$ is odd this number is $\lfloor k l / 2\rfloor$.

Then we subtract those points that we should not have counted. we express this number in terms of the function $\sigma$. The line $y=-x+h+2$ intersects the line $y=l-1$ at $x=h-l+3$. This is the $x$ coordinate of the first point on the last row that is not reachable from $(0, h)$. Then to obtain the number of points that can be reached from this point by moving south-west or north-west, we subtract $h-l+3$ to $k-1$. We have obtained that are $\sigma_{k, l}(k+l-h-4)$ points that we should not have counted.

Example 2. By definition $\sigma_{9,5}(4)$ counts the points in $\mathbf{N}^{2}$ in Table 1 marked with ○. Then $\sigma_{9,5}(4)=9$. Similarly, $\sigma_{9,5}(8)$ counts the points in $\mathbf{N}^{2}$ in Table 1 marked either with the symbol $\circ$ or with the symbol $\bullet$. Then $\sigma_{9,5}(8)=19$.

\begin{tabular}{|l|l|l|l|l|l|l|l|l|}
\hline$\circ$ & & $\circ$ & & $\circ$ & & $\bullet$ & & $\bullet$ \\
\hline & $\circ$ & & $\circ$ & & $\bullet$ & & $\bullet$ & \\
\hline$\circ$ & & $\circ$ & & $\bullet$ & & $\bullet$ & & \\
\hline & $\circ$ & & $\bullet$ & & $\bullet$ & & & \\
\hline$\circ$ & & $\bullet$ & & $\bullet$ & & & & \\
\hline
\end{tabular}

Table 1.

Lemma 3. Let $a, b, c$, and $d$ be in $\mathbf{N}$. Let $R$ be the rectangle with vertices $(a, c)$, $(a+b, c),(a, c+d)$, and $(a+b, c+d)$. We define

$$
\Gamma(a, b, c, d)(x, y)=|\{(u, v) \in R:(x, y) \leadsto(u, v)\}| .
$$


Suppose that $(x, y)$ is such that $x \geq y$. Then

$$
\Gamma(a, b, c, d)(x, y)= \begin{cases}\sigma_{b+1, d+1}(x+y-a-c), & 0 \leq y \leq c \\ \sigma_{b+1, y-c+1}(x-a)+\sigma_{b+1, c+d-y+1}(x-a)-\delta, & c<y<c+d \\ \sigma_{b+1, d+1}(x-y+c+d-a), & c+d \leq y\end{cases}
$$

where $\delta$ is defined as follows If $x<a$, then $\delta=0$. If $a \leq x \leq a+b$, then $\delta=\left\lceil\frac{x-a+1}{2}\right\rceil$. Finally, if $x>a+b$ then we consider two cases: If $x-a-b$ is even then $\delta=\left\lceil\frac{b+1}{2}\right\rceil$; otherwise, $\delta=\left\lfloor\frac{b+1}{2}\right\rfloor$.

Proof. We consider three cases. If $0 \leq y \leq c$ then the first position inside $R$ that we reach is $(x+y-a-c, c)$. Therefore, we assume that we are starting at this point. Similarly, if $y \geq c+d$, then the first position inside $R$ that we reach is $(x-y+c+d-a, c)$. Again, we can assume that we are starting at this point.

On the other hand, if $c<y<c+d$, then we subdivide the problem in two parts. The number of position to the north of us is counted by $\sigma_{b+1, y-c+1}(x-a)$. The number of position to the south of us is counted by $\sigma_{b+1, c+d-y+1}(x-a)$. We define $\delta$ to be the number of points in $\mathbf{N}^{2}$ that we counted twice during this process. Then it is easy to see that $\delta$ is given by the previous definition.

To compute the coefficient $u_{\nu}$ in the expansion $f[X]=\sum_{\eta} u_{\eta} s_{\eta}[X]$ for $f \in \Lambda$, it is enough to expand $f\left[x_{1}+\cdots+x_{n}\right]=\sum_{\eta} u_{\eta} s_{\eta}\left[x_{1}+\cdots+x_{n}\right]$ for any $n \geq l(\nu)$. (See [7, section I.3], for proofs and details.) Therefore, in this section we work with symmetric functions in a finite number of variables.

Let $\mu$ and $\nu$ be two-row partitions. Set $X=1+x$ and $Y=1+y$ in the comultiplication expansion (2) to obtain

$$
s_{\lambda}[(1+y)(1+x)]=\sum_{\mu, \nu} \gamma_{\mu \nu}^{\lambda} s_{\mu}[1+y] s_{\nu}[1+x]
$$

Note that the Kronecker coefficients are zero when $l(\lambda)>4$.

Jacobi's definition of a Schur function on a finite alphabet $s_{\lambda}[X]$ as a quotient of alternants says that

$$
s_{\lambda}[X]=s_{\lambda}\left(x_{1}, \cdots, x_{n}\right)=\frac{\operatorname{det}\left(x_{i}^{\lambda_{j}+n-j}\right)_{1 \leq i, j \leq n}}{\prod_{i<j}\left(x_{i}-x_{j}\right)} .
$$

By the symmetry properties of the Kronecker product it is enough to compute the Kronecker coefficients $\gamma_{\mu \nu}^{\lambda}$ when $\nu_{2} \leq \mu_{2}$.

Theorem 4. Let $\mu, \nu$, and $\lambda$ be partitions of $n$, where $\mu=\left(\mu_{1}, \mu_{2}\right)$ and $\nu=\left(\nu_{1}, \nu_{2}\right)$ are two two-row partitions and let $\lambda=\left(\lambda_{1}, \lambda_{2}, \lambda_{4}, \lambda_{4}\right)$ be a partition of length less than or equal to 4 . Assume that $\nu_{2} \leq \mu_{2}$. Then

$$
\gamma_{\mu \nu}^{\lambda}=(\Gamma(a, b, a+b+1, c)-\Gamma(a, b, a+b+c+d+2, c))\left(\nu_{2}, \mu_{2}+1\right) .
$$


where $a=\lambda_{3}+\lambda_{4}, b=\lambda_{2}-\lambda_{3}, c=\min \left(\lambda_{1}-\lambda_{2}, \lambda_{3}-\lambda_{4}\right)$ and $d=\left|\lambda_{1}+\lambda_{4}-\lambda_{2}-\lambda_{3}\right|$.

Proof. We expand the polynomial $s_{\lambda}[(1+y)(1+x)]=s_{\lambda}(1, y, x, x y)$ in two different ways and obtain the Kronecker coefficients by equating both results. Let $\varphi$ be the polynomial defined by $\varphi=(1-x)(1-y) s_{\lambda}(1, y, x, x y)$. Using Jacobi's definition of a Schur function we obtain

$$
\varphi=\frac{\left|\begin{array}{cccc}
1 & 1 & 1 & 1 \\
y^{\lambda_{1}+3} & y^{\lambda_{2}+2} & y^{\lambda_{3}+1} & y^{\lambda_{4}} \\
x^{\lambda_{1}+3} & x^{\lambda_{2}+2} & x^{\lambda_{3}+1} & x^{\lambda_{4}} \\
(x y)^{\lambda_{1}+3} & (x y)^{\lambda_{2}+2} & (x y)^{\lambda_{3}+1} & (x y)^{\lambda_{4}}
\end{array}\right|}{x y(1-x y)(y-x)(1-x)(1-y)} .
$$

On the other hand, we may use Jacobi's definition to expand $s_{\mu}[1+y]$ and $s_{\nu}[1+x]$ as quotients of alternants. Substitute this into (8):

$$
\begin{aligned}
s_{\lambda}[(1+y)(1+x)] & =\sum_{\substack{\mu=\left(\mu_{1}, \mu_{2}\right) \\
\nu=\left(\nu_{1}, \nu_{2}\right)}} \gamma_{\mu \nu}^{\lambda}\left(\frac{y^{\mu_{2}}-y^{\mu_{1}+1}}{1-y}\right)\left(\frac{x^{\nu_{2}}-x^{\nu_{1}+1}}{1-x}\right) \\
& =\sum_{\substack{\mu=\left(\mu_{1}, \mu_{2}\right) \\
\nu=\left(\nu_{1}, \nu_{2}\right)}} \gamma_{\mu \nu}^{\lambda} \frac{x^{\nu_{2}} y^{\mu_{2}}-x^{\nu_{2}} y^{\mu_{1}+1}-x^{\nu_{1}+1} y^{\mu_{2}}+x^{\nu_{1}+1} y^{\mu_{1}+1}}{(1-x)(1-y)} .
\end{aligned}
$$

Since $\nu_{1}+1$ and $\mu_{1}+1$ are both greater than $\left\lfloor\frac{n}{2}\right\rfloor$, equation (11) implies that the coefficient of $x^{\nu_{2}} y^{\mu_{2}}$ in $\varphi$ is $\gamma_{\mu \nu}^{\lambda}$. It is convenient to define an auxiliary polynomial by

$$
\zeta=(1-x y)(y-x) \varphi .
$$

Let $\xi$ be the polynomial obtained by expanding the determinant appearing in (10). Equations (10) and (12) imply

$$
\zeta=\frac{\xi}{x y(1-x)(1-y)}
$$

Let $\xi_{i, j}$ be the coefficient of $x^{i} y^{j}$ in $\xi$. ( Then $\xi_{i, j}$ is zero if $i \leq 0$ or $j \leq 0$, because $\xi$ is a polynomial divisible by $x y$.) Let $\zeta_{i, j}$ be the coefficient of $x^{i} y^{j}$ in $\zeta$. Then

$$
\sum_{i, j \geq 0} \zeta_{i, j} x^{i} y^{j}=\frac{1}{x y(1-x)(1-y)} \sum_{i, j \geq 0} \xi_{i, j} x^{i} y^{j}=\sum_{i, j, k, l \geq 0} \xi_{i-k, j-l} x^{i-1} y^{j-1} .
$$

Comparing the coefficient of $x^{i} y^{j}$ on both sides of equation (13) we obtain that

$$
\zeta_{i, j}=\sum_{k, l \geq 0} \xi_{i+1-k, j+1-l}=\sum_{k=0}^{i} \sum_{l=0}^{j} \xi_{k+1, l+1}
$$


KRONECKER PRODUCT

We compute $\zeta_{i, j}$ from (14) by expanding the determinant appearing on (10). We consider two cases.

Case 1. Suppose that $\lambda_{1}+\lambda_{4}>\lambda_{2}+\lambda_{3}$. Then

$$
\lambda_{1}+\lambda_{2}+4>\lambda_{1}+\lambda_{3}+3>\lambda_{1}+\lambda_{4}+2 \geq \lambda_{2}+\lambda_{3}+2>\lambda_{2}+\lambda_{4}+1>\lambda_{3}+\lambda_{4} .
$$

We record the values of $\xi_{j+1, i+1}$ in Table 2 . We use the convention that $\xi_{i+1, j+1}$ is zero whenever the $(i, j)$ entry is not in Table 2 .

\begin{tabular}{crrrrrr}
\hline$i \backslash j$ & $\lambda_{3}+\lambda_{4}$ & $\lambda_{2}+\lambda_{4}+1$ & $\lambda_{2}+\lambda_{3}+2$ & $\lambda_{1}+\lambda_{4}+2$ & $\lambda_{1}+\lambda_{3}+3$ & $\lambda_{1}+\lambda_{2}+4$ \\
\hline$\lambda_{3}+\lambda_{4}$ & 0 & -1 & +1 & +1 & -1 & 0 \\
$\lambda_{2}+\lambda_{4}+1$ & +1 & 0 & -1 & -1 & 0 & +1 \\
$\lambda_{2}+\lambda_{3}+2$ & -1 & +1 & 0 & 0 & +1 & -1 \\
$\lambda_{1}+\lambda_{4}+2$ & -1 & +1 & 0 & 0 & +1 & -1 \\
$\lambda_{1}+\lambda_{3}+3$ & +1 & 0 & -1 & -1 & 0 & +1 \\
$\lambda_{1}+\lambda_{2}+4$ & 0 & -1 & +1 & +1 & -1 & 0 \\
\hline
\end{tabular}

Table 2

The values of $\xi_{j+1, i+1}$ when $\lambda_{1}+\lambda_{4} \geq \lambda_{2}+\lambda_{3}$

Equation (14) shows that the value of $\zeta_{i, j}$ can be obtained by adding the entries northwest of the point $(i, j)$. In Table 3 we record the values of $\zeta_{i, j}$.

\begin{tabular}{crrrrrrr}
\hline$i \backslash j$ & $I_{1}$ & $I_{2}$ & $I_{3}$ & $I_{4}$ & $I_{5}$ & $I_{6}$ & $I_{7}$ \\
\hline$I_{1}$ & 0 & 0 & 0 & 0 & 0 & 0 & 0 \\
$I_{2}$ & 0 & 0 & -1 & 0 & +1 & 0 & 0 \\
$I_{3}$ & 0 & +1 & 0 & 0 & 0 & -1 & 0 \\
$I_{4}$ & 0 & 0 & 0 & 0 & 0 & 0 & 0 \\
$I_{5}$ & 0 & -1 & 0 & 0 & 0 & +1 & 0 \\
$I_{6}$ & 0 & 0 & +1 & 0 & -1 & 0 & 0 \\
$I_{7}$ & 0 & 0 & 0 & 0 & 0 & 0 & 0 \\
\hline
\end{tabular}

Table 3

The values of $\zeta_{i, j}$ when $\lambda_{1}+\lambda_{4} \geq \lambda_{2}+\lambda_{3}$

where

$$
\begin{array}{ll}
I_{1}=\left[0, \lambda_{3}+\lambda_{4}\right), & I_{2}=\left[\lambda_{3}+\lambda_{4}, \lambda_{2}+\lambda_{4}\right], \\
I_{3}=\left[\lambda_{2}+\lambda_{4}+1, \lambda_{2}+\lambda_{3}+1\right], & I_{4}=\left[\lambda_{2}+\lambda_{3}+2, \lambda_{1}+\lambda_{4}+1\right], \\
I_{5}=\left[\lambda_{1}+\lambda_{4}+2, \lambda_{1}+\lambda_{3}+2\right], & I_{6}=\left[\lambda_{1}+\lambda_{3}+3, \lambda_{1}+\lambda_{2}+3\right], \\
I_{7}=\left[\lambda_{1}+\lambda_{2}+4, \infty\right) . &
\end{array}
$$

Case 2. Suppose that $\lambda_{1}+\lambda_{4} \leq \lambda_{2}+\lambda_{3}$. Then

$\lambda_{1}+\lambda_{2}+4>\lambda_{1}+\lambda_{3}+3>\lambda_{2}+\lambda_{3}+2>\lambda_{1}+\lambda_{4}+2>\lambda_{2}+\lambda_{4}+1>\lambda_{3}+\lambda_{4}$. 
Note that in Table 3, the rows and columns corresponding to $\lambda_{1}+\lambda_{4}+2$ and $\lambda_{2}+\lambda_{3}+2$ are the same. Therefore, the values of $\xi_{i, j}$ for $\lambda_{1}+\lambda_{4} \leq \lambda_{2}+\lambda_{3}$ are recorded in Table 3 , if we set

$$
\begin{aligned}
& I_{3}=\left[\lambda_{2}+\lambda_{4}+1, \lambda_{1}+\lambda_{4}+1\right] \\
& I_{4}=\left[\lambda_{2}+\lambda_{4}+2, \lambda_{2}+\lambda_{3}+1\right] \\
& I_{5}=\left[\lambda_{2}+\lambda_{3}+2, \lambda_{1}+\lambda_{3}+2\right],
\end{aligned}
$$

and define the other intervals as before.

In both cases, let $\varphi_{i, j}$ be the coefficient of $x^{i} y^{j}$ in $\varphi$. Using (12) we obtain that

$$
\begin{aligned}
\varphi & =\frac{1}{(1-x y)(y-x)} \sum_{i, j \geq 0} \zeta_{i, j} x^{i} y^{j} \\
& =\frac{1}{y-x} \sum_{i, j, l \geq 0} \zeta_{i-l, j-l} x^{i} y^{j} \\
& =\sum_{i, j, k, l \geq 0} \zeta_{i-k-l, j+k-l+1} x^{i} y^{j} .
\end{aligned}
$$

(Note: We can divide by $y-x$ because $\varphi=0$ when $x=y$.) Comparing the coefficients of $x^{i} y^{j}$ on both sides of equation (15), we obtain $\varphi_{i, j}=\sum_{k, l \geq 0} \zeta_{i-k-l, j+k-l+1}$. Therefore,

$$
\varphi_{\nu_{2}, \mu_{2}}=\sum_{i, j=0}^{\nu_{2}} \zeta_{\nu_{2}-i-j, \mu_{2}+i-j+1} .
$$

We have shown that $\gamma_{\mu \nu}^{\lambda}=\varphi_{\nu_{2}, \mu_{2}}$ can be obtained by adding the entries in Table 3 in all points in $\mathbf{N}^{2}$ that can be reached from $\left(\nu_{2}, \mu_{2}+1\right)$. See Table 4.

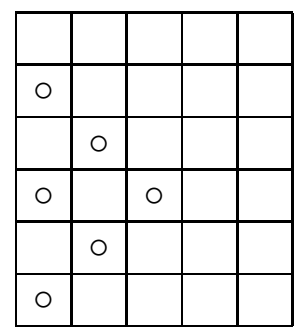

Table 4

The right-most point in Table 4 has coordinates $(3,2)$.

By hypothesis $\nu_{2} \leq \mu_{2} \leq\lfloor n / 2\rfloor$. Then, if we start at $\left(\nu_{2}, \mu_{2}+1\right)$ and move as previously described, the only points in $\mathbf{N}^{2}$ that we can possibly reach and that are nonzero in Table 3 are those in $I_{2} \times I_{3}$ or $I_{2} \times I_{5}$. Hence, we have that $\varphi_{\nu_{2}, \mu_{2}}$ is the number of points in $\mathbf{N}^{2}$ inside $I_{2} \times I_{3}$ that can be reached from $\left(\nu_{2}, \mu_{2}+1\right)$ minus the ones that can be reached in $I_{2} \times I_{5}$. 
Case 1 The inequality $\lambda_{1}+\lambda_{4}>\lambda_{2}+\lambda_{3}$ implies that $\lambda_{1}+\lambda_{4}+1>\left\lfloor\frac{n}{2}\right\rfloor$. Moreover, $\mu_{2} \geq \nu_{2}$ implies that we are only considering the region of $\mathbf{N}^{2}$ given by $0 \leq i \leq j \leq\left\lfloor\frac{n}{2}\right\rfloor$. The number of points in $\mathbf{N}^{2}$ that can be reached from $\left(\nu_{2}, \mu_{2}+1\right)$ inside $I_{2} \times I_{3}$ is given by $\Gamma\left(\lambda_{3}+\lambda_{4}, \lambda_{2}-\lambda_{3}, \lambda_{2}+\lambda_{4}+1, \lambda_{3}-\lambda_{4}\right)$. Similarly, the number of points in $\mathbf{N}^{2}$ that can be reached from $\left(\nu_{2}, \mu_{2}+1\right)$ inside $I_{2} \times I_{5}$ is given by $\Gamma\left(\lambda_{3}+\lambda_{4}, \lambda_{2}-\lambda_{3}, \lambda_{1}+\lambda_{4}+2, \lambda_{3}-\lambda_{4}\right)$.

Case 2 The inequality $\lambda_{2}+\lambda_{3} \geq \lambda_{1}+\lambda_{4}$, implies that $\lambda_{1}+\lambda_{4}+1>\left\lfloor\frac{n}{2}\right\rfloor$. Moreover, $\mu_{2} \geq \nu_{2}$ implies that we are only considering the region of $\mathbf{N}^{2}$ given by $0 \leq i \leq j \leq\left\lfloor\frac{n}{2}\right\rfloor$. The number of points in $\mathbf{N}^{2}$ that can be reached from $\left(\nu_{2}, \mu_{2}+1\right)$ inside $I_{2} \times I_{3}$ is given by $\Gamma\left(\lambda_{3}+\lambda_{4}, \lambda_{2}-\lambda_{3}, \lambda_{2}+\lambda_{4}+1, \lambda_{1}-\lambda_{2}\right)$. Similarly, the number of points in $\mathbf{N}^{2}$ that can be reached from $\left(\nu_{2}, \mu_{2}+1\right)$ inside $I_{2} \times I_{5}$ is given by $\Gamma\left(\lambda_{3}+\lambda_{4}, \lambda_{2}-\lambda_{3}, \lambda_{2}+\lambda_{3}+2, \lambda_{1}-\lambda_{2}\right)$.

Corollary 5. Let $\mu=\left(\mu_{1}, \mu_{2}\right), \nu=\left(\nu_{1}, \nu_{2}\right)$, and $\lambda=\left(\lambda_{1}, \lambda_{2}\right)$ be partitions of $n$. Assume that $\nu_{2} \leq \mu_{2} \leq \lambda_{2}$. Then

$$
\gamma_{\mu \nu}^{\lambda}=(y-x)(y \geq x)
$$

where $x=\max \left(0,\left\lceil\frac{\mu_{2}+\nu_{2}+\lambda_{2}-n}{2}\right\rceil\right)$ and $y=\left\lceil\frac{\mu_{2}+\nu_{2}-\lambda_{2}+1}{2}\right\rceil$.

Proof. Set $\lambda_{3}=\lambda_{4}=0$ in Theorem 4 . Then we notice that the second possibility in the definition of $\Gamma$, that is, when $c<y<c+d$, never occurs. Note that $\nu_{2}+\mu_{2}-\lambda_{2} \geq \nu_{2}+\mu_{2}-\lambda_{1}-1$ for all partitions $\mu, \nu$, and $\lambda$. Therefore,

$$
\gamma_{\mu \nu}^{\lambda}=\sigma_{\lambda_{2}+1,1}\left(\nu_{2}+\mu_{2}-\lambda_{2}\right)-\sigma_{\lambda_{2}+1,1}\left(\nu_{2}+\mu_{2}-\lambda_{1}-1\right)
$$

Suppose that $\nu_{2}+\mu_{2}-\lambda_{2}<0$. From the definition of $\sigma_{\lambda_{2}+1,1}$ we obtain that $\gamma_{\mu \nu}^{\lambda}=0$. Therefore, in order to have $\gamma_{\mu \nu}^{\lambda}$ not equal to zero, we should assume that $\nu_{2}+\mu_{2}-\lambda_{2} \geq 0$.

If $0 \leq \nu_{2}+\mu_{2}-\lambda_{2}<\lambda_{2}+1$, then

$$
\sigma_{\lambda_{2}+1,1}\left(\nu_{2}+\mu_{2}-\lambda_{2}\right)=\left\lceil\frac{\nu_{2}+\mu_{2}-\lambda_{2}+1}{2}\right\rceil
$$

Similarly, if $0 \leq \nu_{2}+\mu_{2}-\lambda_{2}<\lambda_{2}+1$, then

$$
\sigma_{\lambda_{2}+1,1}\left(\nu_{2}+\mu_{2}-\lambda_{1}-1\right)=\left\lceil\frac{\nu_{2}+\mu_{2}+\lambda_{2}-n}{2}\right\rceil
$$

It is easy to see that all other cases on the definition of $\sigma_{k, l}$ can not occur. Therefore, defining $x$ and $y$ as above, we obtain the desired result. 
Example 6. If $\mu=\nu=\lambda=(l, l)$ or $\mu=\nu=(2 l, 2 l)$ and $\lambda=(3 l, l)$, then from the previous corollary, we obtain that

$$
\gamma_{\mu \nu}^{\lambda}=\left\lceil\frac{l+1}{2}\right\rceil-\left\lceil\frac{l}{2}\right\rceil=(l \text { is even })
$$

Note that to apply Corollary 5 to the second family of shapes, we should first use the symmetries of the Kronecker product to set $\nu=\lambda=(2 l, 2 l)$ and $\mu=(3 l, l)$.

Corollary 7. The Kronecker coefficients $\gamma_{\mu \nu}^{\lambda}$, where $\mu$ and $\nu$ are two-row partitions, are unbounded.

Proof. It is enough to construct an unbounded family of Kronecker coefficients. Assume that $\mu=\nu=\lambda=(3 l, l)$. Then from the previous corollary we obtain that

$$
\gamma_{\mu \nu}^{\lambda}=\left\lceil\frac{l+1}{2}\right\rceil
$$

\section{Sergeev's Formula}

In this section we state Sergeev's formula for a Schur function of a difference of two alphabets. See [1] or [7, section I.3] for proofs and comments.

Definition 8. Let $X_{m}=x_{1}+\cdots+x_{m}$ be a finite alphabet, and let $\delta_{m}=(m-1, m-2, \cdots, 1,0)$. We define $X_{m}^{\delta_{m}}$ by $X_{m}^{\delta_{m}}=x_{1}^{m-1} \cdots x_{m-1}$.

Definition 9. Let $i(\alpha)$ denote the number of inversions of the permutation $\alpha$. We define the alternant to be

$$
A_{m}^{x} P=\sum_{\alpha \in S_{m}}(-1)^{i(\alpha)} P\left(x_{\alpha(1)}, \cdots, x_{\alpha(m)}\right),
$$

for any polynomial $P\left(x_{1}, \cdots, x_{n}\right)$.

Definition 10. Let $\Delta$ be the operation of taking the Vandermonde determinant of an alphabet, i.e.,

$$
\Delta\left(X_{m}\right)=\operatorname{det}\left(x_{i}^{m-j}\right)_{i, j=1}^{m}
$$

Theorem 11 (Sergeev's Formula). Let $X_{m}=x_{1}+\cdots+x_{m}$, and $Y_{n}=y_{1}+\cdots+y_{n}$ be two alphabets. Then

$$
s_{\lambda}\left[X_{m}-Y_{n}\right]=\frac{1}{\Delta\left(X_{m}\right) \Delta\left(Y_{n}\right)} A_{m}^{x} A_{n}^{y} X_{m}^{\delta_{m}} Y_{n}^{\delta_{n}} \prod_{(i, j) \in \lambda}\left(x_{i}-y_{j}\right)
$$


The notation $(i, j) \in \lambda$ means that the point $(i, j)$ belongs to the diagram of $\lambda$. We set $x_{i}=0$ for $i>m$ and $y_{j}=0$ for $j>n$.

We use Sergeev's formula as a tool for making some calculations we need for the next two sections.

1. Let $\mu=\left(1^{e_{1}} m_{2}\right)$ be a hook. (We are assuming that $e_{1} \geq 1$ and $m_{2} \geq 2$.) Let $X^{1}=\left\{x_{1}\right\}$ and $X^{2}=\left\{x_{2}\right\}$.

$$
s_{\mu}\left[x_{1}-x_{2}\right]=(-1)^{e_{1}} x_{1}^{m_{2}-1} x_{2}^{e_{1}}\left(x_{1}-x_{2}\right) .
$$

2. Let $\nu=\left(\nu_{1}, \nu_{2}\right)$ be a two-row partition. Let $Y=\left\{y_{1}, y_{2}\right\}$. Then

$$
s_{\nu}\left[y_{1}+y_{2}\right]=\frac{\left(y_{1} y_{2}\right)^{\nu_{2}}\left(y_{1}^{\nu_{1}-\nu_{2}+1}-y_{2}^{\nu_{1}-\nu_{2}+1}\right)}{y_{1}-y_{2}} .
$$

3. We say that a partition $\lambda$ is a double hook if $(2,2) \in \lambda$ and it has the form $\lambda=\left(1^{d_{1}} 2^{d_{2}} n_{3} n_{4}\right)$. In particular any two-row shape is considered to be a double hook.

Let $\lambda$ be a double hook. Let $U=\left\{u_{1}, u_{2}\right\}$ and $V=\left\{v_{1}, v_{2}\right\}$. Then if $n_{4} \neq 0$ then $s_{\lambda}\left[u_{1}+u_{2}-v_{1}-v_{2}\right]$ equals

$$
\begin{aligned}
& \frac{\left(u_{1}-v_{1}\right)\left(u_{2}-v_{1}\right)\left(u_{1}-v_{2}\right)\left(u_{2}-v_{2}\right)}{\left(u_{1}-u_{2}\right)\left(v_{1}-v_{2}\right)}(-1)^{d_{1}}\left(u_{1} u_{2}\right)^{n_{3}-2}\left(v_{1} v_{2}\right)^{d_{2}} \\
& \times\left(u_{2}^{n_{4}-n_{3}+1}-u_{1}^{n_{4}-n_{3}+1}\right)\left(v_{2}^{d_{1}+1}-v_{1}^{d_{1}+1}\right) .
\end{aligned}
$$

On the other hand, if $n_{4}=0$ then to compute $s_{\lambda}\left[u_{1}+u_{2}-v_{1}-v_{2}\right]$ we should write $\lambda$ as $\left(1^{d_{1}} 2^{d_{2}-1} 2 n_{3}\right)$. That is, we set $d_{1}:=d_{1}, d_{2}:=d_{2}-1, n_{3}:=2$, and $n_{4}=n_{3}$ in (19).

4. Let $\lambda$ be a hook shape, $\lambda=\left(1^{d_{1}} n_{2}\right)$. (We are assuming that $d_{1} \geq 1$ and $n_{2} \geq 2$.) Let $U=\left\{u_{1}, u_{2}\right\}$ and $V=\left\{v_{1}, v_{2}\right\}$. Then $s_{\lambda}\left[u_{1}+u_{2}-v_{1}-v_{2}\right]$ equals

$$
\begin{aligned}
& (-1)^{d_{1}-1} \frac{1}{\left(u_{1}-u_{2}\right)} \frac{1}{\left(v_{1}-v_{2}\right)} \times \\
& \left\{u_{1} v_{1}\left(u_{1}-v_{1}\right)\left(u_{1}-v_{2}\right)\left(u_{2}-v_{1}\right) u_{1}^{n_{2}-2} v_{1}^{d_{1}-1}\right. \\
& \quad-u_{1} v_{2}\left(u_{1}-v_{2}\right)\left(u_{1}-v_{1}\right)\left(u_{2}-v_{2}\right) u_{1}^{n_{2}-2} v_{2}^{d_{1}-1} \\
& \quad-u_{2} v_{1}\left(u_{2}-v_{1}\right)\left(u_{2}-v_{2}\right)\left(u_{1}-v_{1}\right) u_{2}^{n_{2}-2} v_{1}^{d_{1}-1} \\
& \left.\quad+u_{2} v_{2}\left(u_{2}-v_{2}\right)\left(u_{2}-v_{1}\right)\left(u_{1}-v_{2}\right) u_{2}^{n_{2}-2} v_{2}^{d_{1}-1} .\right\}
\end{aligned}
$$




\section{The CASE OF TWO HOOK SHAPES}

In this section we derive an explicit formula for the Kronecker coefficients $\gamma_{\mu \nu}^{\lambda}$ in the case in which $\mu=\left(1^{e} u\right)$, and $\nu=\left(1^{f} v\right)$ are both hook shapes. Given a partition $\lambda$ the Kronecker coefficient $\gamma_{\mu \nu}^{\lambda}$ tells us whether point $(u, v)$ belongs to some regions in $\mathbf{N}^{2}$ determined by $\mu, \nu$ and $\lambda$.

Recall that we denote the characteristic function by enclosing a proposition $P$ with brackets, $(P)$.

Lemma 12. Let $(u, v) \in \mathbf{N}^{2}$ and let $R$ be the rectangle with vertices $(a, b),(b, a)$, $(c, d)$, and $(d, c)$, with $a \geq b, c \geq d, c \geq a$ and $d \geq b$. (Sometimes, when $c=d=e$, we denote this rectangle as $(a, b ; e)$.)

Then $(u, v) \in R$ if and only if $|v-u| \leq a-b$ and $a+b \leq u+v \leq c+d$

Proof. Let $L_{1}$ be the line of slope 1 passing through $(u, v)$, and let $L_{2}$ be the line of slope -1 passing through $(u, v)$. Then we have that

$$
\begin{aligned}
& L_{1}: y=x+v-u \\
& L_{2}: y=-x+u+v
\end{aligned}
$$

The point $(u, v)$ is in $R$ if and only if $L_{1}$ is between the lines of slope 1 passing throught $(a, b)$ and $(b, a)$. That is, $a-b \leq v-u \leq b-a$ and $L_{2}$ is between the lines of slope -1 passing throught $(a, b)$ and $(c, d)$. That is, $a+b \leq u+v \leq c+d$

Theorem 13. Let $\lambda, \mu$ and $\nu$ be partitions of $n$, where $\mu=\left(1^{e} u\right)$ and $\nu=\left(1^{f} v\right)$ are hook shapes. Then the Kronecker coefficients $\gamma_{\mu \nu}^{\lambda}$ are given by the following:

1. If $\lambda$ is a one-row shape, then $\gamma_{\mu \nu}^{\lambda}=\delta_{\mu, \nu}$.

2. If $\lambda$ is not contained in a double hook shape, then $\gamma_{\mu \nu}^{\lambda}=0$.

3. Let $\lambda=\left(1^{d_{1}} 2^{d_{2}} n_{3} n_{4}\right)$ be a double hook. Let $x=2 d_{2}+d_{1}$. Then

$$
\begin{aligned}
\gamma_{\mu \nu}^{\lambda}=\left(n_{3}-1 \leq\right. & \left.\frac{e+f-x}{2} \leq n_{4}\right)\left(|f-e| \leq d_{1}\right) \\
& +\left(n_{3} \leq \frac{e+f-x+1}{2} \leq n_{4}\right)\left(|f-e| \leq d_{1}+1\right) .
\end{aligned}
$$

Note that if $n_{4}=0$, then we shall rewrite $\lambda=\left(1^{d_{1}} 2^{d_{2}-1} 2 n_{3}\right)$ before using the previous formula.

4. Let $\lambda=\left(1^{d} w\right)$ be a hook shape. Suppose that $e \leq u, f \leq v$, and $d \leq w$. Then

$$
\gamma_{\mu \nu}^{\lambda}=(e \leq d+f)(d \leq e+f)(f \leq e+d)
$$

Proof. Set $X=\{1, x\}$ and $Y=\{1, y\}$ in the comultiplication expansion (2) to obtain

$$
s_{\lambda}[(1-x)(1-y)]=\sum_{\mu, \nu} \gamma_{\mu \nu}^{\lambda} s_{\mu}[1-x] s_{\nu}[1-y]
$$


We use equation (17) to replace $s_{\mu}$ and $s_{\nu}$ in the right hand side of (21). Then we divide the resulting equation by $(1-x)(1-y)$ to get

$$
\frac{s_{\lambda}[1-y-x+x y]}{(1-x)(1-y)}=\sum_{\mu, \nu} \gamma_{\mu \nu}^{\lambda}(-x)^{e}(-y)^{f} .
$$

Therefore,

$$
\gamma_{\mu \nu}^{\lambda}=\left[(-x)^{e}(-y)^{f}\right] \frac{s_{\lambda}[1-y-x+x y]}{(1-x)(1-y)},
$$

when $\mu$ and $\nu$ are hook shapes.

Case 1. If $\lambda$ is not contained in any double hook, then the point $(3,3)$ is in $\lambda$, and by Sergeev's formula, $s_{\lambda}[1-y-x+x y]$ equals zero.

Case 2. Let $\lambda=\left(1^{d_{1}} 2^{d_{2}} n_{3} n_{4}\right)$ be a double hook. Set $u_{1}=1, u_{2}=x y, v_{1}=x$, and $v_{2}=y$ in $(19)$. Then we divide by $(1-x)(1-y)$ on both sides of the resulting equation to obtain

$$
\begin{aligned}
\frac{s_{\lambda}[1-y-x+x y]}{(1-x)(1-y)} & =(-1)^{d_{1}}(x y)^{n_{3}+d_{2}-1} \\
& \times(1-x)(1-y)\left(\frac{1-(x y)^{n_{4}-n_{3}+1}}{1-x y}\right)\left(\frac{x^{d_{1}+1}-y^{d_{1}+1}}{x-y}\right) .
\end{aligned}
$$

Note: If $n_{4}=0$ then we should write $\lambda=\left(1^{d_{1}} 2^{d_{2}-1} 2 n_{4}\right)$ in order to use (19).

Let $p$ be a point in $\mathbf{N}^{2}$. We say that $(i, j)$ can be reached from $p$, written $p \leadsto$ $(i, j)$, if $(i, j)$ can be reached from $p$ by moving any number of steps south-west or north-west. We have defined a weight function by

$$
\omega_{p}(i, j)= \begin{cases}x^{i} y^{j}, & \text { if } p \leadsto(i, j) ; \\ 0, & \text { otherwise. }\end{cases}
$$

Let $\omega_{p}(T)=\sum_{(i, j) \in T} \omega_{p}(i, j)$ be the generating function of a region $T$ in $\mathbf{N}^{2}$. Let $R$ be the rectangle with vertices $\left(0, d_{1}\right),\left(d_{1}, 0\right),\left(d_{1}+n_{4}-n_{3}, n_{4}-n_{3}\right)$ and $\left(n_{4}-n_{3}, d_{1}+n_{4}-n_{3}\right)$. Then

$$
\begin{aligned}
\omega_{\left(d_{1}+n_{4}-n_{3}, n_{4}-n_{3}\right)}(R) & =\left(\frac{1-(x y)^{n_{4}-n_{3}+1}}{1-x y}\right)\left(\frac{x^{d_{1}+1}-y^{d_{1}+1}}{x-y}\right) \\
& =\sum_{k=0}^{n_{4}-n_{3}} \sum_{i+j=d_{1}}(x y)^{k} x^{i} y^{j} .
\end{aligned}
$$

See Table 5. 


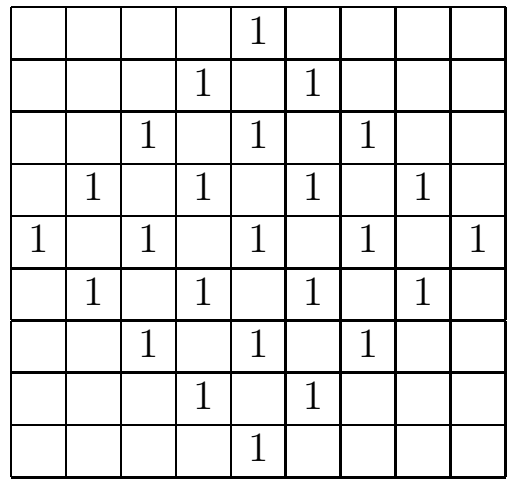

Table 5 .

$$
d_{1}=4 \text { and } n_{3}-n_{4}=4
$$

Recall that we are using matrix coordinates, and that the upper-left corner has coordinates $(0,0)$. The coordinates of the four vertices of $R$ in Table 5 are $(0,4)$, $(4,0),(8,4)$, and $(4,8)$.

We interpret the right-hand side of (22) as the sum of four different generating functions. To be more precise, the right-hand side of (22) can be written as $\sum_{i=1}^{4} \omega_{p_{i}}\left(r_{i}\right)$ where $p_{1}=\left(n_{4}+d_{2}-1, n_{4}+d_{2}+d_{1}-1\right)$ and $R_{1}=\left\{n_{3}+d_{2}+d_{1}-1, n_{3}+\right.$ $\left.d_{2}-1 ; n_{4}-n_{3}\right\}, p_{2}=\left(n_{4}+d_{2}, n_{3} 4+d_{2}+d_{1}-1\right)$ and $R_{2}=\left\{n_{3}+d_{2}+d_{1}, n_{3}+d_{2}-\right.$ $\left.1 ; n_{4}-n_{3}\right\}, p_{3}=\left(n_{4}+d_{2}-1, n_{4}+d_{2}+d_{1}\right)$ and $R_{3}=\left\{n_{3}+d_{2}+d_{1}-1, n_{3}+d_{2} ; n_{4}-n_{3}\right\}$, and $p_{4}=\left(n_{4}+d_{2}+d_{1}, n_{4}+d_{2}+d_{1}\right)$ and $R_{4}=\left\{n_{3}+d_{2}+d_{1}, n_{3}+d_{2}+d_{1} ; n_{4}-n_{3}\right\}$.

We observe that $R_{1} \cup R_{2}$ (and $R_{3} \cup R_{4}$ ) are rectangles in $\mathbf{N}^{2}$. Moreover,

$$
\gamma_{\mu \nu}^{\lambda}=\left((e, f) \in R_{1} \cup R_{2}\right)+\left((e, f) \in R_{3} \cup R_{4}\right) .
$$

The vertices of rectangle $R_{1} \cup R_{4}$ are given (using the notation of 12) by

$$
\begin{array}{lll}
a=n_{3}+d_{2}+d_{1}-1 & b=n_{3}+d_{2}-1 \\
c=n_{4}+d_{2}+d_{1} & d=n_{4}+d_{2}
\end{array}
$$

Similarly, the vertices of rectangle $R_{2} \cup R_{3}$ are given by

$$
\begin{array}{ll}
a=n_{3}+d_{2}+d_{1} & b=n_{3}+d_{2}-1 \\
c=n_{4}+d_{2}+d_{1} & d=n_{4}+d_{2}-1
\end{array}
$$

Applying Lemma 12 to (23) we obtain

$$
\begin{aligned}
\gamma_{\mu \nu}^{\lambda}=\left(n_{3}-1 \leq\right. & \left.\frac{e+f-x}{2} \leq n_{4}\right)\left(|f-e| \leq d_{1}\right) \\
& +\left(n_{3} \leq \frac{e+f-x+1}{2} \leq n_{4}\right)\left(|f-e| \leq d_{1}+1\right) .
\end{aligned}
$$


KRONECKER PRODUCT

Case 3. $\lambda$ is a hook. Suppose that $\lambda$ is a hook, $\lambda=\left(1^{d} w\right)$. Set $u_{1}=1$, $u_{2}=x y, v_{1}=x$, and $v_{2}=y$ in (20). Then we divide by $(1-x)(1-y)$ on both sides of the resulting equation to obtain

$$
\begin{aligned}
& \frac{s_{\lambda}[1-y-x+x y]}{(1-x)(1-y)}=(-1)^{d}\left(\frac{x^{d+1}-y^{d+1}}{x-y}\right)\left(\frac{1-(x y)^{w}}{1-x y}\right) \\
& \quad+(-1)^{d-1} x y\left(\frac{x^{d}-y^{d}}{x-y}\right)\left(\frac{1-(x y)^{w-1}}{1-x y}\right) .
\end{aligned}
$$

We want to interpret this equation as a generating function for a region $T$ using the weight $\omega$. We proceed as follows:

Let $R_{1}$ be the rectangle with vertices $(d, 0),(0, d),(d+w-1, w-1)$, and $(w-1, d+w-1)$. Then

$$
\omega_{(w-1, d+w-1)}\left(R_{1}\right)=\left(\frac{1-(x y)^{w}}{1-x y}\right)\left(\frac{x^{d+1}-y^{d+1}}{x-y}\right)=\sum_{k=0}^{w-1} \sum_{i+j=d}(x y)^{k} x^{i} y^{j} .
$$

(See Table 5.) Similarly, let $R_{2}$ be the rectangle with vertices $(d, 1),(1, d),(d+$ $w-2, w-1)$, and $(w-1, d+w-2)$. Then

$$
\omega_{(w-1, d+w-2)}\left(R_{2}\right)=x y\left(\frac{1-(x y)^{w-1}}{1-x y}\right)\left(\frac{x^{d}-y^{d}}{x-y}\right)=x y \sum_{k=0}^{w-2} \sum_{i+j=d-1}(x y)^{k} x^{i} y^{j} .
$$

Observe that the points in $\mathbf{N}^{2}$ that can be reached from $(0, d)$ in $R_{1}$ and the points in $\mathbf{N}^{2}$ that can be reached from $(1, d)$ in $R_{2}$ are disjoint. Moreover, they completely fill the rectangle $R_{1} \cup R_{2}$. See Table 6 .

\begin{tabular}{|l|r|r|r|r|r|r|r|r|r|}
\hline & & & & 1 & & & & & \\
\hline & & & 1 & -1 & 1 & & & & \\
\hline & & 1 & -1 & 1 & -1 & 1 & & & \\
\hline & 1 & -1 & 1 & -1 & 1 & -1 & 1 & & \\
\hline 1 & -1 & 1 & -1 & 1 & -1 & 1 & -1 & 1 & \\
\hline & 1 & -1 & 1 & -1 & 1 & -1 & 1 & -1 & 1 \\
\hline & & 1 & -1 & 1 & -1 & 1 & -1 & 1 & \\
\hline & & & 1 & -1 & 1 & -1 & 1 & & \\
\hline & & & & 1 & -1 & 1 & & & \\
\hline & & & & & 1 & & & & \\
\hline
\end{tabular}

Table 6

$d=4, w=6$. 
Note that $R_{2}$ is contained in $R_{1}$. We obtain that

$$
\omega_{(w-1, d+w-1)}\left(R_{1}\right)+\omega_{(w-1, d+w-2)}\left(R_{2}\right)=\left|(e, f) \in R_{1}\right|
$$

We use apply Lemma 12 to the previous equation to obtain:

$$
(|e-f| \leq d)(d \leq e+f \leq d+2 w-2) .
$$

But, by hypothesis, $e \leq u, f \leq v$, and $d \leq w$. Therefore, this system is equivalent to $(d \leq e+f)(f \leq e+d)(e \leq d+f)$, as desired.

Corollary 14. Let $\lambda, \mu$, and $\nu$ be partitions of $n$, where $\mu=\left(1^{e} u\right)$ and $\nu=$ $\left(1^{f} v\right)$ are hook shapes and $\lambda=\left(\lambda_{1}, \lambda_{2}\right)$ is a two-row shape. Then the Kronecker coefficients $\gamma_{\mu \nu}^{\lambda}$ are given by

$$
\gamma_{\mu \nu}^{\lambda}=\left(\lambda_{2}-1 \leq e \leq \lambda_{1}\right)(e=f)+\left(\lambda_{2} \leq \frac{e+f+1}{2} \leq \lambda_{1}\right)(|e-f| \leq 1) .
$$

Proof. In Theorem 13, set $d_{1}=d_{2}=0, n_{3}=\lambda_{2}$ and $n_{4}=\lambda_{1}$.

Corollary 15. Let $\lambda, \mu$ and $\nu$ be partitions of $n$, where $\mu$ and $\nu$ are hook shapes. Then the Kronecker coefficients are bounded. Moreover, the only possible values for the Kronecker coefficients are 0, 1 or 2.

\section{The CASE OF A HOOK Shape AND A TWO-ROW SHAPE}

In this section we derive an explicit formula for the Kronecker coefficients in the case $\mu=\left(1^{e_{1}} m_{2}\right)$ is a hook and $\nu=\left(\nu_{1}, \nu_{2}\right)$ is a two-row shape. Given a partition $\lambda$, the Kronecker coefficients $\gamma_{\mu \nu}^{\lambda}$ tell us whether the point $\left(e_{1}, \nu_{2}\right)$ belongs to some regions in $\mathbf{N}^{2}$ determined by $\mu, \nu$ and $\lambda$.

Using the symmetry properties of the Kronecker product, we may assume that if $\lambda=\left(1^{d_{1}} 2^{d_{2}} n_{3} n_{4}\right)$ then $n_{4}-n_{3} \leq d_{1}$. (If $n_{4}=0$ then we should rewrite $\lambda$ as $\left(1^{d_{1}} 2^{d_{2}-1} 2 n_{3}\right)$. Moreover, our hypothesis becomes $n_{3}-2 \leq d_{1}$.)

Recall that we denote the value of the characteristic function at proposition $P$ by $(P)$.

Theorem 16. Let $\lambda, \mu$ and $\nu$ be partitions of $n$, where $\mu=\left(1^{e_{1}} m_{2}\right)$ is a hook and $\nu=\left(\nu_{1}, \nu_{2}\right)$ is a two-row shape. Then the Kronecker coefficients $\gamma_{\mu \nu}^{\lambda}$ are given by the following:

1. If $\lambda$ is a one-row shape, then $\gamma_{\mu \nu}^{\lambda}=\delta_{\mu, \nu}$.

2. If $\lambda$ is not contained in any double hook, then $\gamma_{\mu \nu}^{\lambda}=0$. 
3. Suppose $\lambda=\left(1^{d_{1}} 2^{d_{2}} n_{3} n_{4}\right)$ is a double hook. Assume that $n_{4}-n_{3} \leq d_{1}$. (If $n_{4}=0$, then we should write $\lambda=\left(1^{d_{1}} 2^{d_{2}-1} 2 n_{3}\right)$.) Then

$$
\begin{aligned}
\gamma_{\mu \nu}^{\lambda}=\left(n_{3} \leq\right. & \left.\nu_{2}-d_{2}-1 \leq n_{4}\right)\left(d_{1}+2 d_{2}<e_{1}<d_{1}+2 d_{2}+3\right) \\
& +\left(n_{3} \leq \nu_{2}-d_{2} \leq n_{4}\right)\left(d_{1}+2 d_{2} \leq e_{1} \leq d_{1}+2 d_{2}+3\right) \\
+ & \left(n_{3} \leq \nu_{2}-d_{2}+1 \leq n_{4}\right)\left(d_{1}+2 d_{2}<e_{1}<d_{1}+2 d_{2}+3\right) \\
& \quad-\left(n_{3}+d_{2}+d_{1}=\nu_{2}\right)\left(d_{1}+2 d_{2}+1 \leq e_{1} \leq d_{1}+2 d_{2}+2\right)
\end{aligned}
$$

4. If $\lambda$ is a hook, see Corollary 14 .

Proof. Set $X=1+x$ and $Y=1+y$ in the comultiplication expansion (2) to obtain

$$
s_{\lambda}[(1-x)(1+y)]=\sum_{\mu, \nu} \gamma_{\mu \nu}^{\lambda} s_{\mu}[1-x] s_{\nu}[1+y]
$$

Use (17) and (18) to replace $s_{\mu}$ and $s_{\nu}$ in the right-hand side of (27), and divide by $(1-x)$ to obtain

$$
\frac{s_{\lambda}[(1-x)(1+y)]}{1-x}=\sum_{\substack{\mu=\left(1^{e_{1}} m_{2}\right) \\ \nu=\left(\nu_{1}, \nu_{2}\right)}} \gamma_{\mu \nu}^{\lambda}(-x)^{e_{1}} y^{\nu_{2}}\left(\frac{1-y^{\nu_{1}-\nu_{2}+1}}{1-y}\right) .
$$

If $\lambda$ is not contained in any double hook, then the point $(3,3)$ is in $\lambda$, and by Sergeev's formula, $s_{\lambda}[(1-x)(1+y)]$ equals zero.

Since we already computed the Kronecker coefficients when $\lambda$ is contained in a hook, we can assume for the rest of this proof that $\lambda$ is a double hook. Let $\lambda=\left(1^{d_{1}} 2^{d_{2}} n_{3} n_{4}\right)$. (Note: If $n_{4}=0$ then we should write $\lambda=\left(1^{d_{1}} 2^{d_{2}-1} 2 n_{4}\right)$.)

Set $u_{1}=1, u_{2}=y, v_{1}=x$, and $v_{2}=x y$ in (19), and multiply by $\frac{1-y}{1-x}$ on both sides of the resulting equation.

$$
\begin{aligned}
& \sum_{\substack{\mu=\left(1^{e_{1}} m_{2}\right) \\
\nu=\left(\nu_{1}, \nu_{2}\right)}} \gamma_{\mu \nu}^{\lambda}(-x)^{e_{1}} y^{\nu_{2}}\left(1-y^{\nu_{1}-\nu_{2}+1}\right)=(y-x)(1-x y)(1-x) \\
& \quad \times(-x)^{d_{1}+2 d_{2}} y^{n_{3}+d_{2}-1}\left(\frac{\left(1-y^{n_{4}-n_{3}+1}\right)\left(1-y^{d_{1}+1}\right)}{1-y}\right) .
\end{aligned}
$$

We have that $(y-x)(1-x y)(1-x)=y-x\left(1+y+y^{2}\right)+x^{2}\left(1+y+y^{2}\right)-x^{3} y$. Therefore, looking at the coefficient of $x$ on both sides of the equation, we see that $\gamma_{\mu \nu}^{\lambda}$ is zero if $e_{1}$ is different from $d_{1}+2 d_{2}, d_{1}+2 d_{2}+1, d_{1}+2 d_{2}+2$, or $d_{1}+2 d_{2}+3$. 
Let $e_{1}=d_{1}+2 d_{2}$ or $e_{1}=d_{1}+2 d_{2}+3$. Since $\nu_{2} \leq n / 2$, we have that

$$
\begin{aligned}
\gamma_{\mu \nu}^{\lambda} & =\left[y^{\nu_{2}}\right] \sum_{\substack{\mu=\left(1^{e_{1}} m_{2}\right) \\
\nu=\left(\nu_{1}, \nu_{2}\right)}} \gamma_{\mu \nu}^{\lambda} y^{\nu_{2}} & \\
& =\left[y^{\nu_{2}}\right] \sum_{\substack{\mu=\left(1^{e_{1}} m_{2}\right) \\
\nu=\left(\nu_{1}, \nu_{2}\right)}} \gamma_{\mu \nu}^{\lambda} y^{\nu_{2}}\left(1-y^{\nu_{1}-\nu_{2}+1}\right) & \left(\nu_{1}+1>n / 2\right) \\
& =\left[y^{\nu_{2}}\right] y^{n_{3}+d_{2}}\left(1-y^{d_{1}+1}\right)\left(\frac{1-y^{n_{4}-n_{3}+1}}{1-y}\right) & \quad \text { Eq. 29] }) \\
& =\left[y^{\nu_{2}}\right] y^{n_{3}+d_{2}}\left(1-y^{d_{1}+1}\right) \sum_{k=0}^{n_{4}-n_{3}} y^{k} & \\
& =\left[y^{\nu_{2}}\right] y^{n_{3}+d_{2}} \sum_{k=0}^{n_{4}-n_{3}} y^{k} . & \left(n_{3}+d_{2}+d_{1} \geq n / 2\right)
\end{aligned}
$$

We have obtained that for $e_{1}=d_{1}+2 d_{2}$ or $e_{1}=d_{1}+2 d_{2}+3$

$$
\gamma_{\mu \nu}^{\lambda}=\left(n_{3} \leq \nu_{2}-d_{2} \leq n_{4}\right)
$$

Let $e_{1}=d_{1}+2 d_{2}+1$ or $e_{1}=d_{1}+2 d_{2}+2$. Since $\nu_{2} \leq\left\lfloor\frac{n}{2}\right\rfloor$ we have that

$$
\begin{aligned}
\gamma_{\mu \nu}^{\lambda} & =\left[y^{\nu_{2}}\right] \sum_{\substack{\mu=\left(1^{\left.e_{1} m_{2}\right)} \\
\nu=\left(\nu_{1}, \nu_{2}\right)\right.}} \gamma_{\mu \nu}^{\lambda} y^{\nu_{2}} \\
& =\left[y^{\nu_{2}}\right] \sum_{\substack{\mu=\left(1^{e_{1}} m_{2}\right) \\
\nu=\left(\nu_{1}, \nu_{2}\right)}} \gamma_{\mu \nu}^{\lambda}\left(1-y^{\nu_{1}-\nu_{2}+1}\right) \\
& =\left[y^{\nu_{2}}\right] y^{n_{3}+d_{2}-1}\left(1+y+y^{2}\right)\left(1-y^{d_{1}+1}\right)\left(\frac{1-y^{n_{4}-n_{3}+1}}{1-y}\right) \\
& =\left(\left[y^{\nu_{2}}\right] y^{n_{3}+d_{2}-1}\left(1+y+y^{2}\right)\left(\frac{1-y^{n_{4}-n_{3}+1}}{1-y}\right)\right)-\left(n_{3}+d_{2}+d_{1}=\nu_{2}\right) \\
& =\left(\left[y^{\nu_{2}}\right] y^{n_{3}+d_{2}-1}\left(1+y+y^{2}\right) \sum_{k=0}^{n_{4}-n_{3}} y^{k}\right)-\left(n_{3}+d_{2}+d_{1}=\nu_{2}\right)
\end{aligned}
$$

We have obtained that for $e_{1}=d_{1}+2 d_{2}+1$ or $e_{1}=d_{1}+2 d_{2}+2$

$$
\begin{aligned}
\gamma_{\mu \nu}^{\lambda}=\left(n_{3} \leq \nu_{2}-d_{2}-1 \leq\right. & \left.n_{4}\right)+\left(n_{3} \leq \nu_{2}-d_{2} \leq n_{4}\right) \\
& +\left(n_{3} \leq \nu_{2}-d_{2}+1 \leq n_{4}\right)-\left(n_{3}+d_{2}+d_{1}=\nu_{2}\right)
\end{aligned}
$$


Corollary 17. The Kronecker cofficients, $\gamma_{\mu \nu}^{\lambda}$, where $\mu$ is a hook and $\nu$ is a tworow shape are always $0,1,2$ or 3 .

\section{Final COMments}

The inner product of symmetric functions was discovered by J. H. Redfield [8] in 1927, together with the scalar product of symmetric functions. He called them cup and cap products, respectively. D.E. Littlewood [5, 6] reinvented the inner product in 1956 .

More recently, I.M. Gessel [3] and A. Lascoux [4] obtained combinatorial interpretations for the Kronecker coefficients in some restricted cases; Lascoux in the case where $\mu$ and $\nu$ are hooks, and $\lambda$ a straight tableaux, and Gessel in the case that $\mu$ and $\nu$ are zigzag shapes and $\lambda$ is an arbitrary skew shape. A. Lascoux interpreted the Kronecker coefficients, when two of the shapes are hooks as counting clases of words under some equivalence relation. We refer to [4] or [2] for a complete statement of his results. The Corollary of Theorem 3 in this paper shows that each class of words, under Lascoux's equivalence, contains either 0 , 1, or 2 different representatives. I. Gessel worked on a more general framework, contemplating the occurrence of skew tableaux. It was shown in [2] that in the case where two of the partitions are hook shapes, and the third one is an arbitrary straight shape, his result is equivalent to Lascoux's.

In [2], A.M. Garsia and J.B. Remmel founded a way to relate shuffles of permutations and Kronecker coefficients. From here they obtained a combinatorial interpretation for the Kronecker coefficients when $\lambda$ is a product of homogeneous symmetric functions, and $\mu$ and $\nu$ are arbitrary skew shapes. They also showed how Gessel's and Lascoux's results are related.

J.B. Remmel [9], [10], and J.B. Remmel and T. Whitehead [11, obtained formulas for computing the Kronecker coefficients in the same cases considered in this paper. Their approach was mainly combinatorial:

First, they expanded the Kronecker product $s_{\mu} * s_{\nu}$ in terms of Schur functions using the Garsia-Remmel algorithm [2]. The problem of computing the Kronecker coefficients was reduced to computing signed sums of certain products of skew Schur functions.

Then they obtained a description of the coefficients that arise in the expansion of the resulting product of skew Schur functions in terms of counting 3-colored diagrams in [9], and [10] or 4-colored diagrams in [11]. At this point, they reduced the problem to computing a signed sum of colored diagram.

Finally, they defined involutions on these signed sums to cancel negative terms, and obtained the desired formulas by counting classes of restricted colored diagram.

In general, it is not obvious how to go from the determination of the Kronecker coefficients $\gamma_{\mu, \nu}^{\lambda}$ when $\mu$ and $\nu$ are two-row shapes found in this paper, and the one 
obtained by J.B. Remmel and T. Whitehead [11]. But, in some particular cases this is easy to see. For instance, when $\lambda$ is also a two-row shape, both formulas are exactly the same.

\section{REFERENCES}

[1] N. Bergeron and A.M. Garsia, "Sergeev's Formula and the Littlewood-Richardson Rule," Linear and Multilinear Algebra 27, 1990, pp. 79-100.

[2] A.M. Garsia and J.B. Remmel, "Shuffles of permutations and Kronecker products," Graphs Combin. 1985, pp. 217-263.

[3] I.M. Gessel, "Multipartite P-partitions and inner products of Schur functions," Contemp. Math. 1984, pp. 289-302.

[4] A. Lascoux, "Produit de Kronecker des representations du group symmetrique," Lecture Notes in Mathematics 1980, 795, Springer Verlag pp. 319-329.

[5] D.E. Littlewood, "The Kronecker product of symmetric group representations," J. London Math. Soc. 31, 1956, pp. 89-93.

[6] D.E. Littlewood, "Plethysm and inner product of S-functions," J. London Math. Soc. 32, 1957, pp. 18-22.

[7] I.G. Macdonald, Symmetric Functions and Hall Polynomials, second edition, Oxford University Press, 1995.

[8] J.H. Redfield "The theory of group reduced distribution," Amer. J. Math. 49, 1927, pp. 433-455.

[9] J.B. Remmel, "A formula for the Kronecker product of Schur functions of hook shapes," $J$. Algebra 120, 1989, pp. 100-118.

[10] J.B. Remmel, "Formulas for the expansion of the Kronecker products $S_{(m, n)} \otimes S_{\left(1^{p-r}, r\right)}$ and $S_{\left(1^{k} 2^{l}\right)} \otimes S_{\left(1^{p-r}, r\right)}, "$ Discrete Math. 99, 1992, pp. 265-287.

[11] J.B. Remmel and T. Whitehead, "On the Kronecker product of Schur functions of two row shapes," Bull. Belg. Math. Soc. Simon Stevin 1, 1994, pp. 649-683.

[12] B.E. Sagan, The Symmetric Group, Wadsworth \& Brooks/Cole, Pacific Grove, California, 1991.

Department of Mathematics, Brandeis University, Waltham, MA 02254

E-mail address: rosas@math.brandeis.edu 